Randomized Experimental Trial

\title{
Pulsed Radiofrequency Improves Neuropathic Pain in Chronic Constriction Injury Rats through the Upregulation of the Transcription and Translation Levels of Glial Cell Line-Derived Neurotrophic Factor
}

\author{
Jin Hailong, MD1, Ren Hao, MD¹, Jia Zipu, MD¹, Ji Nan, MD², and Luo Fang, MD¹
}

\footnotetext{
From: ${ }^{1}$ Department of Anesthesiology and Pain

Management, Beijing Tiantan Hospital, Capital Medical University, Beijing,

P.R. China; ${ }^{2}$ Department

of Neurosurgery, Beijing

Tiantan Hospital, Capital Medical University, Beijing, P.R. China

Address Correspondence: Luo Fang, MD Department of Anesthesiology and Pain Management, Beijing Tiantan Hospital, Capital Medical University

Beijing 100050, P.R. China E-mail:

luofangwt@yahoo.com

Disclaimer: See pg. 40. Conflict of interest: Each author certifies that he or she, or a member of his or her immediate family, has no commercial association

(i.e., consultancies,

stock ownership, equity interest, patent/licensing arrangements, etc.) that might pose a conflict of interest in connection with the submitted manuscript.

Manuscript received: o9$13-2016$

Revised manuscript received: 05-03-2017

Accepted for publication: 06-27-2017

Free full manuscript: www.painphysicianjournal.
}

com
Background: Several studies in recent years have confirmed that the direct application of pulsed radiofrequency (PRF) on peripheral nerve compression points can alleviate hyperalgesia in animal models of neuropathic pain (NP). However, the mechanism underlying the treatment of peripheral nerves by PRF is unclear.

Objectives: We aim to observe changes in pain behavior after the application of PRF on the ligation site of the sciatic nerves (SNs) of rats with chronic constriction injury $(\mathrm{CCl})$ and to investigate the effects of PRF on the transcription and translation levels of glial cell line-derived neurotrophic factor (GDNF) in nerve tissues at the treatment site.

\section{Study Design: A randomized, experimental trial}

Setting: Experimental Animal Center, Beijing Tiantan Hospital.

Methods: Ninety-six adult male Sprague-Dawley rats were randomly divided into 4 groups: sham-sham (SS) group, sham-PRF (SP) group, CCI-sham (CS) group, and CCI-PRF (CP) group. The right SNs of rats in the CS and $\mathrm{CP}$ group were ligated to establish the $\mathrm{CCI}$ model. The right SNs in the SS and SP groups were isolated and exposed but without being ligated. On the fourteenth day after $\mathrm{CCl} /$ sham operation, PRF treatment was performed on the midpoint of the ligation sites of the SN in the CP group and the corresponding sites in the SP group. The electrode was only placed at the ligation sites of the SN in the CS group and the corresponding sites in the SS group without current being applied. The 50\% paw withdrawal threshold (50\%PWT) and thermal withdrawal latency (TWL) of rats in all of the groups were measured. The transcription and translation levels of GDNF of the PRF/sham treatment sites were measured before and after treatment by reverse transcription-polymerase chain reaction (RT-PCR) and enzyme-linked immunosorbent assays (ELISAs).

Results: The 50\%PWT value of the hind paws of rats in the CP group gradually increased on day 6 after the PRF treatment and was significantly higher than that in the CS group ( 6 days after treatment $P<0.05 ; 14$ days after treatment $P<0.01$ ). The TWL value in the CP group was higher than that in the CS group 2 days after treatment $(P<0.05)$ and was significantly higher $(P<0.01)$ from day 6 until the end of the experiment. On the day 6 and 14 after PRF treatment, the mRNA and protein expression levels of GDNF at the ligation sites of the SNs of rats in the CP group were higher than both the levels before treatment and those in the CS group $(P<0.01)$.

Limitations: The efficacy of PRF treatment in the CCI model was only tested within 14 days, and the changes in GDNF levels were only tested at 3 time-points before and after treatment.

Conclusions: The direct application of PRF on SN ligation sites in the CCI model can safely and effectively alleviate NP. One of the mechanisms of this effect could be the upregulation of the transcription and translation of GDNF in compressed SNs.

Key words: Pulsed radiofrequency, chronic constriction injury, sciatic nerve, 50\% paw withdrawal threshold, thermal withdrawal latency, glia cell line-derived neurotrophic factor, reverse transcription-polymerase chain reaction, enzyme-linked immunosorbent assay

Pain Physician 2018; 21:33-40 
$\mathbf{N}$ europathic pain (NP) can be divided into peripheral and central types. Different types of NP have similar or common pathogenic mechanisms, including peripheral sensitization, central sensitization, and dysfunction of the descending inhibitory system (1). The current status of clinical treatment of NP is unsatisfactory. Refractory NP that does not respond to conservative treatment, such as drugs, should be treated with minimally invasive techniques or even surgical treatment.

Pulsed radiofrequency (PRF) is a type of non-destructive and minimally invasive treatment. This technique was first proposed by Sluijter et al (2) in 1997, and its most significant advantage is that there is no damage to neural tissues as a result of treatment. A series of animal studies have reported the efficacies and mechanisms of PRF application to the dorsal root ganglia (DRG) in a variety of peripheral NP models $(3,4)$. In recent years, a small number of researchers began to notice that the direct application of PRF on peripheral nerves at the compression sites of NP rats could also effectively alleviate NP $(5,6)$. Some clinical observation studies and case reports have confirmed that PRF can effectively alleviate NP in humans. However, the treatment guidelines of peripheral nerves using PRF have not been established, and the mechanism of action is still unclear.

NP is characterized by spontaneous burning pain, hyperalgesia, and allodynia. The presentations of the chronic constriction injury $(\mathrm{CCl})$ model are very similar to the clinical presentations of human peripheral NP. Therefore, this injury model is commonly used to investigate the efficacy of novel treatment methods and the possible mechanisms of action (7). Our previous studies showed that the pain behavior of rats and the histopathological prognosis of injured nerves were significantly improved after 14 days of direct PRF application on sciatic nerve (SN) ligation sites in a $\mathrm{CCl}$ model. In addition, the protein level of glial cell line-derived neurotrophic factor (GDNF) was found to be significantly upregulated (8). GDNF is an important neurotrophic factor that presents in the central and peripheral nervous systems, exhibiting nutritional, protective, and repair-promoting functions after nerve injury $(9,10)$. Therefore, we hypothesized that PRF might promote the self-repair of injured peripheral nerves and alleviate NP through the upregulation of GDNF protein levels. However, the mechanism of PRF relieves NP by upregulated GDNF is unclear. A better understanding of this mechanism requires further analysis of the changes in the transcription and translation levels of GDNF at dif- ferent time-points before and after the PRF treatment and the relationship of these effects with the changes in pain behaviors.

\section{Methods}

\section{Experimental Animals}

Male Sprague-Dawley rats at the age of 4 months with a body weight of $200-220 \mathrm{~g}$ were selected. The housing environment was $22-24^{\circ} \mathrm{C}$, with food and water provided ad libitum and a 12-hour light-dark cycle. This study was approved by the Beijing Tiantan Hospital Experimental Animal Welfare Ethics Committee.

\section{Experimental Groups}

A total of 96 rats were randomly divided into 4 groups ( $n=24$ in each group): (1) sham-sham group (SS), (2) sham-PRF group (SP), (3) CCl-sham group (CS), and (4) $\mathrm{CCl}$-PRF group (CP). The baseline pain-related values of rats in the 4 groups were measured and collected before the $\mathrm{CCl} /$ sham operation. Multiple measurements were performed on days 2, 6, 10, and 14 after the $\mathrm{CCl} /$ sham operation and the PRF/sham treatment. The time-points for collection of the SN trunks at the ligation sites of rats in the CS and CP groups or the corresponding sites in the SS and SP groups were before the PRF/sham treatment and on days 6 and 14 after treatment (Table 1).

\section{Induction of NP and PRF Treatment}

Rats in the CS and CP groups were used for establishment of the $\mathrm{CCl}$ model according to the method of Bennett and Xie (11). The right SN was exposed after anesthesia and 4 ligatures ( 4 - 0 chromic catgut) were placed at the proximal end of the trifurcations. The width between each ligation was $1 \mathrm{~mm}$, and the strength was appropriate for inducing mild twitching of the calf muscle.

On the fourteenth day after the $\mathrm{CCl} /$ sham operation, the CP and SP groups received PRF treatment. The SN ligation sites or corresponding sites were exposed again, along the original incision. The PRF electrode (PMF-21-50-2, Baylis Medical Inc., Montreal, Canada) was vertically placed at the midpoint of the ligation site or at the corresponding sites of the SNs. The parameter settings of the RF therapeutic instrument (PMG-230, Baylis Medical Inc., Montreal, Canada) were as follows (5): $2 \mathrm{~Hz}, 42^{\circ} \mathrm{C}, 45 \mathrm{~V}$, and last for 180 seconds. The electrode was fixed in the SS and CS groups for 180 seconds without the application of electric current. 
Pulsed Radiofrequency Improves Neuropathic Pain Through the Upregulation of GDNF

Table 1. Experimental procedure of rats in all of the groups at different time-points.

\begin{tabular}{||l|c|c|c|c|c|c|c||}
\hline \multirow{3}{*}{ Group } & \multicolumn{7}{|c|}{ Time-Points } \\
\cline { 2 - 8 } & Day 0 & Days 2/6/10 & Day 14 & $\begin{array}{c}\text { Day 16 } \\
\text { (day } \mathbf{2} \text { after } \\
\text { PRF/sham } \\
\text { treatment) }\end{array}$ & $\begin{array}{c}\text { Day 20 } \\
\text { (day 6 after } \\
\text { PRF/sham } \\
\text { treatment) }\end{array}$ & $\begin{array}{c}\text { Day 24 } \\
\text { (day 10 after } \\
\text { PRF/sham } \\
\text { treatment) }\end{array}$ & $\begin{array}{c}\text { Day 28 } \\
\text { (day 14 after } \\
\text { PRF/sham } \\
\text { treatment) }\end{array}$ \\
\hline SS & Behavioral test\# & Behavioral test & $\begin{array}{c}\text { Behavioral test\# } \\
\text { Tissue collection }\end{array}$ & Behavioral test & Behavioral test\# & Behavioral test & Behavioral test\# \\
\hline SP & Sham operating & & $\begin{array}{c}\text { Sham/PRF } \\
\text { treatment }\end{array}$ & & Tissue collection & & Tissue collection \\
\hline CS & Behavioral test\# & Behavioral test & $\begin{array}{c}\text { Behavioral test\# } \\
\text { Tissue collection }\end{array}$ & Behavioral test & Behavioral test\# & Behavioral test & Behavioral test\# \\
\hline CP & CCI procedure & & $\begin{array}{c}\text { Sham/PRF } \\
\text { treatment }\end{array}$ & & Tissue collection & & Tissue collection \\
\hline
\end{tabular}

\# Behavioral tests were performed on days 0 and 14 of the experiment before the CCI/sham surgery and the PRF/sham treatment, as well as on days 14,20 , and 28 before tissue collection.

\section{Behavioral Studies}

\section{0\% Paw Withdrawal Threshold (PWT) Test (12)}

The experiment used 8 Von Frey hairs (VFHs) (Stoelting Portal, Kiel, WI) with logarithmically increasing strength $(0.41,0.70,1.20,2.04,3.63,5.50,8.51$, and $15.14 \mathrm{~g}$ ) to vertically stimulate the right hind paw of rats. The stimulation began at $2.04 \mathrm{~g}$, with a slight bending of the probe and holding for $2-3$ seconds; the duration of stimulation was 30 seconds. Brisk withdrawal of paws was recorded as a positive reaction. If the reaction was negative, VFH stimulation with the next strongest VFH was performed. If the paw withdrawal reaction was positive, the next weakest VFH was used for stimulation. The negative reaction preceding the first positive reaction was used as the starting point; a total of 6 continuous stimulations, including the starting point, were performed. The following formula was used: $50 \%$ PWT $=10^{(\mathrm{Xf}+\times \delta) / 104}\left(\mathrm{X}_{\mathrm{f}}\right.$ : the logarithmic value of the last VFH in the sequence; $\kappa$ : obtained using a table of values, based on the measured sequence; $\delta=0.224$ ) (13).

\section{Thermal Withdrawal Latency (TWL) Test}

The rats were placed in separated transparent cages, with the bottom being a glass plate. The rats were pre-adapted for 30 minutes. The heating center of an infrared radiant heat stimulus generator (7371, Ugo Basile, Comerio, Italy) was directed at the right hind paw of rats for continuous radiation. The latency time between the start of the heating to the reflexive withdrawal of hind paw due to the heat was measured. The measurement was performed 3 times, and the mean value was recorded (8).

\section{GDNF Expression Analysis}

\section{Reverse Transcription-Polymerase Chain Reaction (RT-PCR)}

On days 14,20 , and 28 of the experiment, the rats were sacrificed by deep anesthesia ( $n=8$ for each timepoint in each group). The $\mathrm{SN}$ trunks at the ligation sites and the corresponding sites were collected. Each specimen was divided into 2 samples each. One of the samples was randomly chosen for RT-PCR analysis. 1) Total RNA in tissues samples was extracted using TRIzol (Invitrogen) and stored at $-80^{\circ} \mathrm{C}$. 2) CDNA synthesis using reverse transcription was performed according to the instructions of the MMLV first strand synthesis reagent kit (NEB). The extracted RNA was used to obtain first strand cDNA. RNA $(6 \mu \mathrm{l})$ and Oligo $(\mathrm{dT})_{18}(0.5 \mu \mathrm{g} / \mu \mathrm{l} ; 2 \mu \mathrm{l})$ were first added with RNase-free $\mathrm{dH}_{2} \mathrm{O}$ to a total volume of $10 \mu \mathrm{l}$. The sample was heated to $70^{\circ} \mathrm{C}$ for $5 \mathrm{~min}$ utes and then rapidly placed on ice for 2 minutes. Next, the $20 \mu$ l reaction mix $(5 \times$ reaction buffer, $10 \mathrm{mM}$ dNTP Mix, Primescript II RNase, RNase inhibitor, and RNasefree $\mathrm{dH}_{2} \mathrm{O}$ ) was placed in a $42^{\circ} \mathrm{C}$ water bath for 1 hour. Lastly, the reverse transcriptase was denatured and deactivated at $85^{\circ} \mathrm{C}$ for 5 minutes. The obtained final RT solution was used as the CDNA solution. 3) For real-time quantitative PCR detection, using the gene sequence information in GenBank, primers appropriate for the SYBR green method were designed using the Primer3 primer design software. $\beta$-actin was used as the internal control (primer sequences are shown in Table 2). The PCR reaction system $(20 \mu$ reaction system) was as follows: Fast SYBR Green MasterMix $(10 \mu \mathrm{l})$, upstream primer $(0.5 \mu \mathrm{l})$, downstream primer $(0.5 \mu \mathrm{l})$, RNase-free 
Pain Physician: January/February 2018; 21:33-40

Table 2. Primers used for real-time polymerase chain reaction (RT-PCR).

\begin{tabular}{|c|c|c|c||}
\hline Gene & GenBank Numbers & Product Length & Sequences (5' - 3') \\
\hline Glia cell line-derived neurotrophic factor & \multirow{2}{*}{ NM_019139 } & \multirow{2}{*}{$172 \mathrm{bp}$} & Forward: ATGAAGTTATGGGATGTCGTGG \\
\cline { 1 - 2 }$($ GDNF) & \multirow{2}{*}{ NM_031144 } & \multirow{2}{*}{$150 \mathrm{bp}$} & Reverse: GATAATCTTCGGGCATATTGGA \\
\cline { 1 - 1 } Beta-actin & & Forward: CCCATCTATGAGGGTTACG \\
\hline ( $\beta$-actin $)$ & & Reverse: TTTAATGTCACGCACGATT \\
\hline
\end{tabular}

$\mathrm{dH}_{2} \mathrm{O}(7 \mu \mathrm{l})$, and CDNA $(2 \mu \mathrm{l})$. The reaction conditions were as follows: $95^{\circ} \mathrm{C}$ pre-denaturation for 20 seconds, $95^{\circ} \mathrm{C}$ denaturation for 30 seconds, $60^{\circ} \mathrm{C}$ annealing for 30 seconds, and $72^{\circ} \mathrm{C}$ extension for 15 seconds, with 40 cycles of amplification. The analysis was performed using a real-time fluorescence quantitative PCR system (ABI step one plus, Applied Biosystems, Foster City, CA). The reliability of the RT-PCR reaction was determined based on the melting curve and the standard curve. The measured threshold cycle was obtained. Using $\beta$-actin as the reference, the obtained threshold value was used to calculate the relative GDNF mRNA expression level using the $2^{-\Delta \Delta C T}$ method (3 technical replicates were used for each sample, and the mean value of the results was obtained).

\section{Enzyme-Linked Immunosorbent Assay (ELISA)}

The other half of the sample was washed with normal saline, dried using filter paper, weighed, homogenized, and centrifuged at $2500 \mathrm{rpm}$ for $1 \mathrm{~min}-$ ute. The supernatant was stored in a $-70^{\circ} \mathrm{C}$ freezer for subsequent detection analysis. The supernatant was processed strictly according to the instruction of the ELISA reagent kit (Elabscience Biotechnology Co., Ltd, Wuhan, China). The optical density (OD) value at 450 $\mathrm{nm}$ was measured using a microplate reader (BIO-RAD 680, Bio-Rad, Hercules, CA). Lastly, according to the OD value of the sample, the corresponding GDNF level was determined on the curve graph. The GDNF protein concentrations in the SN trunks of rats in all of the groups were calculated (8).

\section{Statistical Analysis}

The statistical analysis of experimental data was performed using SPSS Version 18.0 (SPSS Inc., Chicago, IL). The measurement data are expressed as the mean \pm standard deviation $(X \pm S D)$. The $50 \%$ PWT and TWL results were compared using repeated measures analysis of variance (ANOVA). The GDNF mRNA and protein levels in the nerve tissues of rats in all groups were compared using one-way ANOVA, and $\mathrm{S}-\mathrm{N}-\mathrm{K}$ test was used in post hoc analysis. $P<0.05$ indicated statistical significance, and $P<0.01$ indicated a very significant difference.

\section{Results}

\section{0\%PWT}

The baseline values of $50 \%$ PWT of the 4 groups were not different before the experiment $(P>0.05)$. After the $\mathrm{CCl}$ operation, the $50 \%$ PWT gradually decreased for the hind paw on the ligation side in the CS and CP group rats. On day 14 after surgery, the difference was significant compared to the SS group $(P<$ 0.001). At the end of the experiment, the $50 \%$ PWT in the CS group was still significantly lower than in the SS group $(P<0.001)$. Starting from day 6 after the PRF treatment, the $50 \%$ PWT of the CP group was gradually increased and significantly higher than CS group $(P<$ 0.001). At the end of the experiment, the $50 \%$ PWT was still significantly higher than the CS group $(P<0.001)$ but was lower than SS group $(P<0.01, P=0.008)$. Rats in the SP group only exhibited a slight increase in the $50 \%$ PWT on the second day after PRF intervention; this effect was significant compared to the SS group $(P<$ $0.05, P=0.017$ ) and was returned to the normal level after 4 days. The $50 \%$ PWT in the SS group did not change significantly throughout the experimental period $(P>$ 0.05) (Fig. 1).

\section{TWL}

The TWLs of the 4 groups were not different prior to the experiment $(P>0.05)$. Starting from the second day after model establishment, the TWLs of the hind paw on the ligation side of the CS and CP groups were significantly reduced. On the fourteenth day after operation, the TWLs were significantly lower in the CS and CP groups than in the SS group $(P<0.001)$. The TWL in the CS group after sham treatment was significantly lower than in the SS group at all time-points $(P<0.001)$. The TWL in the CP group after the PRF treatment gradually increased; on the second day after treatment, the TWL in the CP group was higher than in the CS group $(P<0.05, P=0.021)$. Beginning on the sixth day after 
treatment, the TWL in the CP group was significantly higher than in the CS group $(P<0.001)$. Moreover, this effect was maintained until the end of the experiment. The TWL in the SP group was slightly higher than in the SS group only on the second day after the PRF intervention $(P<0.05, P=0.044)$, which returned to baseline levels after 4 days. The TWL in the SS group did not change during the experiment $(P>0.05)$ (Fig. 2).

\section{RT-PCR}

Before the PRF/sham treatment, GDNF mRNA levels were significantly increased in the CS and CP groups compared to the SS group $(P<0.001)$. The difference between the SP and SS groups did not reach statistical significance $(P>0.05)$. On the sixth day after PRF treatment, GDNF mRNA in the CP group significantly increased compared to the levels that were observed before treatment $(P<0.001)$. Up to the fourteenth day after treatment, GDNF mRNA levels were still higher than before treatment $(P<0.001)$ and were significantly higher than in the CS group $(P<0.01, P=0.015)$. The GDNF mRNA levels in the CS group before and after the sham treatment were not significantly different $(P$ $>0.05$ ). The GDNF mRNA expression levels in the SP and SS groups did not significantly change during the experiment $(P>0.05)$ (Fig. 3).

\section{ELISA}

The GDNF protein levels at the SN ligation site in the CS and CP groups were significantly increased before the PRF/sham treatment compared to the SS group $(P<0.001)$. However, the difference between the CS and $\mathrm{CP}$ group did not reach statistical significance $(P>$ 0.05). GDNF protein levels in the CP group on the sixth day after PRF treatment were significantly increased compared to before treatment $(P<0.001)$. Up to the fourteenth day after treatment, GDNF protein levels in the CP group were higher than before treatment ( $P$ $<0.001)$ and were significantly higher than in the CS group $(P<0.001)$. GDNF protein levels in the CS group did not change significantly before and after treatment $(P>0.05)$. The differences in GDNF expression levels between the SP and SS groups did not reach statistical significance during the experiment $(P>0.05)$ (Fig. 4).

\section{Discussion}

After SN ligation in this study, the 50\% PWT and TWL of rats in the CS and CP groups consistently, gradually, and significantly decreased compared to the SS group. These results showed that the hind paw at the li-
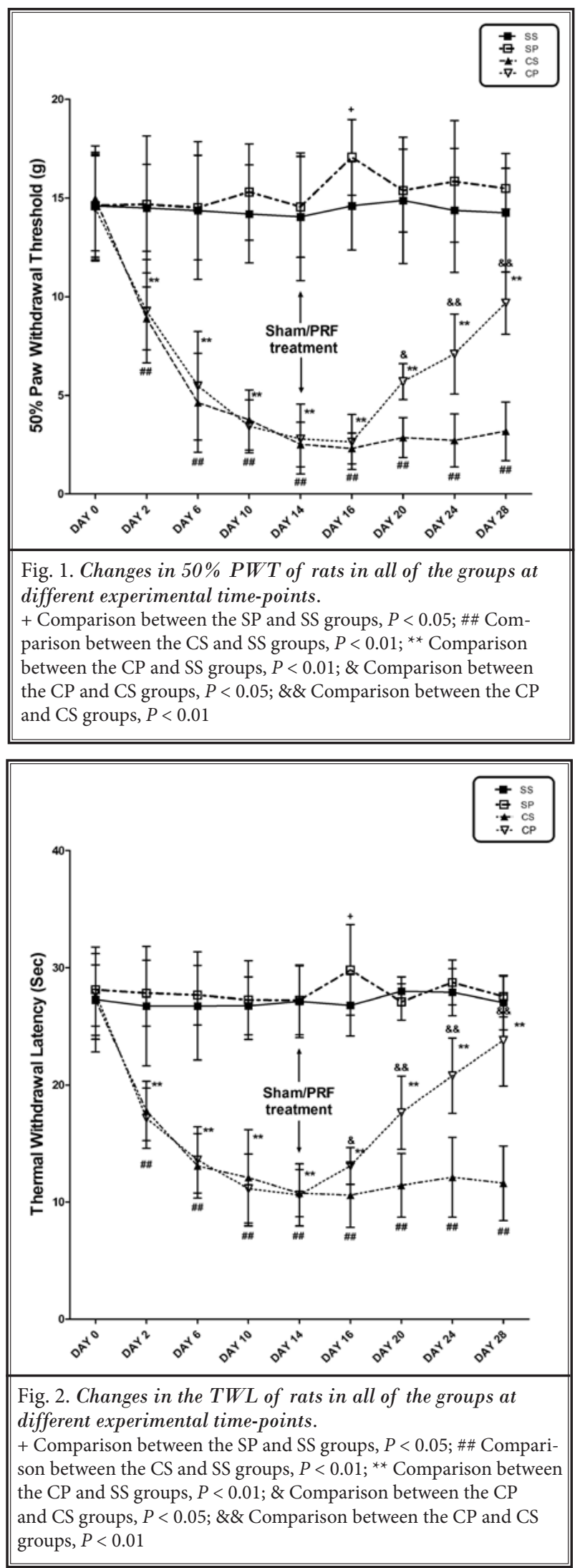


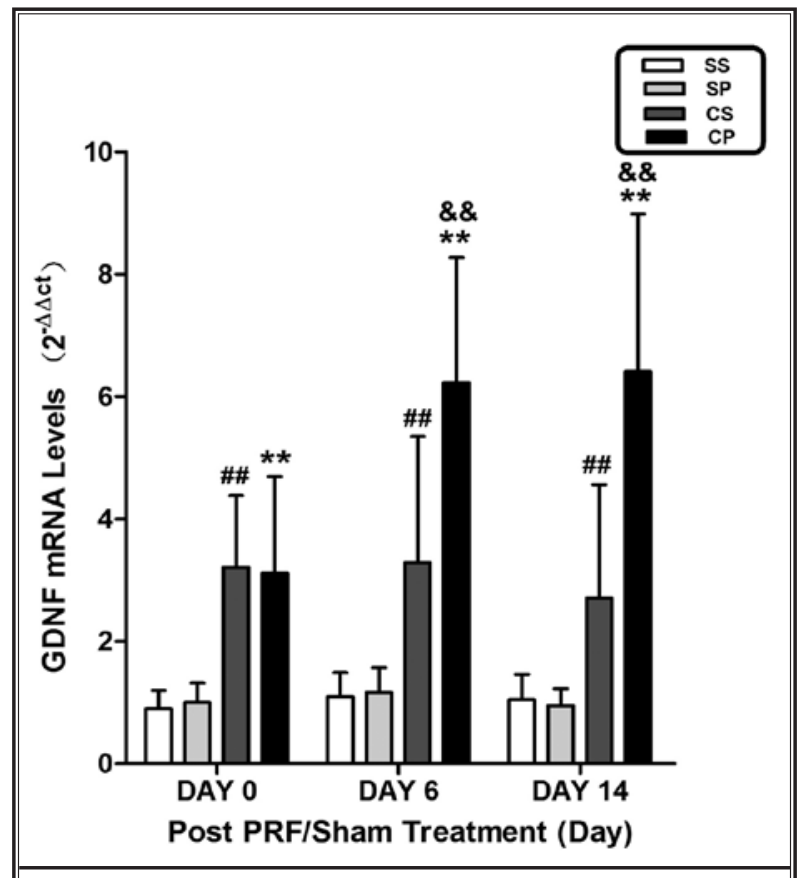

Fig. 3. Changes in GDNF $m R N A$ levels at the $S N$ ligation sites of rats in all of the groups at different experimental time-points.

\#\# Comparison between the CS and SS groups, $P<0.01$; ${ }^{* *}$ Comparison between the CP and SS groups, $P<0.01 ; \& \&$ Comparison between the $\mathrm{CP}$ and CS groups, $P<0.01$

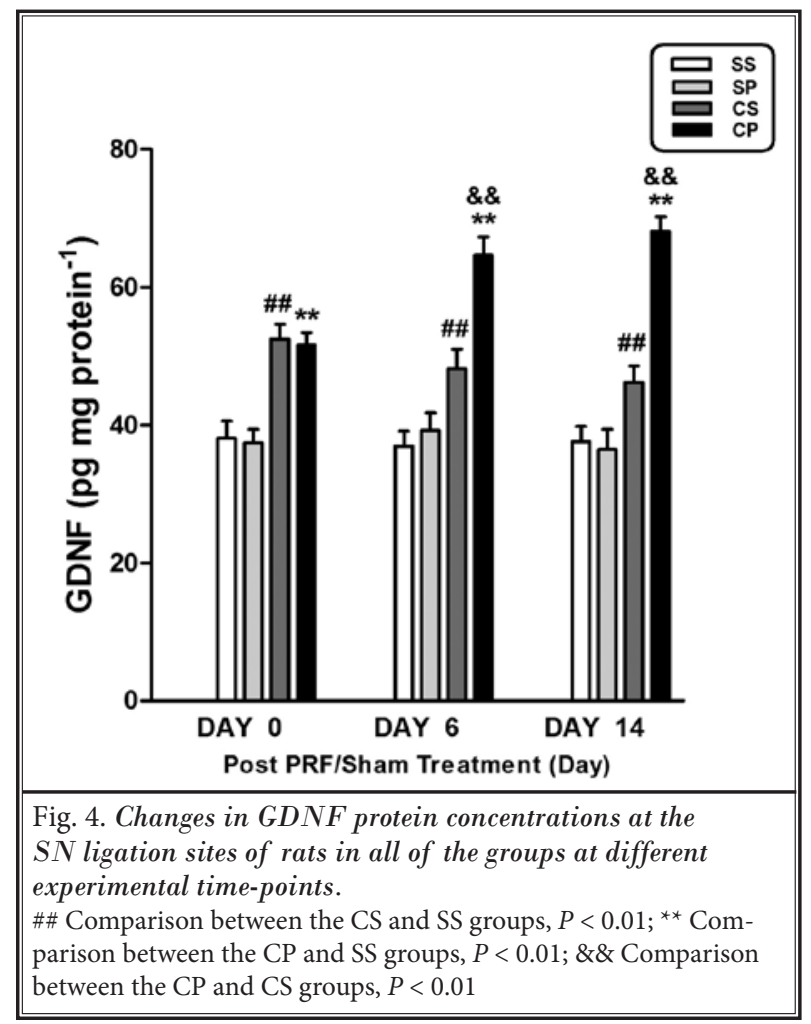

gation side gradually exhibited mechanical and thermal hyperalgesia, indicating that the model was established successfully. The PRF electrode was directly applied on the SN ligation site of the rats in the CP group and the corresponding site in the SP group. On the first 6 days after treatment, the mechanical hyperalgesia of rats in the CP group did not improve, but the thermal hyperalgesia slightly improved. Starting from the sixth day, mechanical hyperalgesia and thermal hyperalgesia both significantly improved, and this effect was maintained until the end of the experiment. It's interesting that the thermal hyperalgesia was reduced 4 days earlier than the mechanical hyperalgesia after PRF treatment in this study for reasons unknown, while most previously published literatures reported that the thermal and mechanical hyperalgesia were relieved almost simultaneously $(6,8)$. Furthermore, PRF treatment helped more in thermal than in mechanical hyperalgesia in this study. These results confirmed that the direct application of PRF on the compressed peripheral nerve trunk was effective for NP treatment. However, on the fourteenth day after treatment, upon completion of the experiment, neither the mechanical nor thermal hyperalgesia of rats in the CP group had completely recovered to the control levels in the SS group; specifically, these values recovered to $67.9 \%$ and $88.0 \%$ of the levels in the SS group, respectively, suggesting that the efficacy of treatment of peripheral NP using PRF requires further improvement. Whether the efficacy can be increased through other combinating treatment methods or by adjusting the pulse parameters will require more studies. In the present study, starting from the sixth day after the PRF treatment to the end of the experiment, mechanical and thermal hyperalgesia of the rats in the CP group showed continuous improvement, suggesting that future studies should extend the observation period to 1) investigate whether PRF can completely alleviate NP and 2) determine the duration of the analgesic effect of PRF. It is worth noting that the alleviation of hyperalgesia by PRF began approximately 6 days after treatment. Therefore, we hypothesize that PRF might exert its effects in such a way that the alleviation of NP is gradually achieved. Currently, the treatment guidelines of PRF on peripheral nerve in clinical practice have not been clarified. Although the NP models can mimic the human NPs to a certain degree, it's difficult to speculate how many times PRF treatment in humans are needed to achieve analgesic effects and for how long that could last, which need in-depth randomized control studies in the future. Similarly, Vallejo et al (5) also 
observed that the effect of PRF began on approximately the fourth day after treatment when directly applied PRF on peripheral nerves of rats in a spared nerve injury (SNI) model. The results of this study suggest that direct treatment of the peripheral nerve trunk by PRF in the clinic might not have immediate analgesic effects. Therefore, appropriate symptomatic treatment should be performed before PRF begins to exert its effect. In spite of this, the results of this study were inspiring, while no current pharmacological option can provide even close results in NP. Further studies may be required to translate the encouraging results into clinical practice in patients undergoing refractory NP.

Early animal experiment studies indicated that the treatment of a variety of peripheral NP models using PRF on the DRG was clearly effective $(14,15)$. Recent studies showed that directly applied PRF to the peripheral nerve compression site could also alleviate NP symptoms. Obviously, DRG puncture is a more complex procedure than peripheral nerve puncture; in addition, the former method usually requires treating several segments, resulting in more trauma and heightened risks. Therefore, treatment of the peripheral nerve by PRF may have greater clinical prospects. However, the best target for treatment of NP using PRF in the clinic is not known. Studies are required that use the same NP model and therapeutic parameters but different therapeutic targets to detect any differences in efficacy.

GDNF was first purified and obtained from rat glioma cells in 1993 by Lin et al (16). It is an important neurotrophic factor that can affect neuronal survival, growth, and directed differentiation. Currently, an increasing amount of evidence indicates that GDNF might play an important role in the transduction and regulation of pain signals, possibly at the NP stage $(17,18)$. Our previous studies showed that PRF might alleviate NP through the upregulation of GDNF translation. In the present study, GDNF expression levels at the SN ligation sites of the $\mathrm{CP}$ and $\mathrm{CS}$ group rats were significantly higher than in the SS group on the fourteenth day after model establishment. This result was consistent with previous reports of GDNF upregulation after chronic peripheral nerve injury $(19,20)$. It is currently considered that the upregulation of GDNF expression in local nerve tissues might promote the self-repair of nerves after peripheral nerve injury (8). However, the observed level of upregulation has been limited and did not result in the complete alleviation of NP. In this study, the GDNF, mRNA, and protein levels of rats in the CP group were all significantly higher on the sixth and fourteenth days after PRF treatment than those before treatment. These levels were also significantly higher than in the CS group that received the sham treatment. The changes in GDNF were consistent with changes in mechanical and thermal hyperalgesia that were observed in the rats after treatment. We could only speculate from the results in this study that one of the mechanisms underlying the treatment of NP in $\mathrm{CCl}$ rats by PRF might involve the upregulation of local transcription and translation levels of GDNF in compressed SNs.

Recent studies have indicated that increased GDNF expression in an NP model using transgenic technology (21) or exogenous administration (22) can significantly alleviate NP. In-depth studies should be performed to determine whether a combination of the above methods to further increase GDNF expression in an NP model results in synergistic effects in the PRF-mediated treatment of peripheral nerves.

\section{Limitations}

However, this study still had some limitations. First, we only observed the efficacy of treatment of the $\mathrm{CCl}$ model using PRF over 14 days, the long term efficacy of this method should be studied further. Secondly, we only detected changes in GDNF at 3 time-points before and after treatment and only tested the SN instead of other locations in the pain loop for changes in GDNF expression. Moreover, we did not antagonize GDNF production and subsequently observe the efficacy of PRF to clarify the mechanism; other possible mechanisms were not investigated. For example whether the downstream GDNF effectors phosphoinositide 3-kinase (PI3K) and AKT signaling would be regulated or not to support the idea of cell survival and self-renewal promoted by PRF treatment in this model. Furthermore, the effect of changing the parameters of PRF treatment, such as the frequency and the output voltage, should be considered. To increase the efficacy of PRF on the peripheral NP, in-depth studies should be performed to select the ideal therapeutic targets and parameters and to develop rational combination therapy strategies.

\section{Conclusions}

In summary, the direct application of PRF on SN ligation sites safely and effectively alleviated NP caused by the $\mathrm{CCl}$ operation. One of the mechanisms of this effect might involve the upregulation of the transcription and translation of GDNF in compressed local nerve tissues. 


\section{Disclaimer}

This study was supported by the Foundation for The Excellent Medical Staff of Beijing (No. 2011-3-034 and No. 2014-3-035). Jin Hailong and Ren Hao contrib- uted equally to this work. Luo Fang and Ji Nan contributed equally to this work in designing and supervising the project.

\section{References}

1. Campbell JN, Meyer RA. Mechanisms of neuropathic pain. Neuron 2006; 52:77-92.

2. Sluijter ME. Non-thermal radiofrequency procedures in the treatment spinal pain. Pain in Europe; Barcelona: 2nd Annual Congress of the European Federation of IASP Chapters. 1997; 326.

3. Laboureyras E, Rivat C, Cahana A, Richebé P. Pulsed radiofrequency enhances morphine analgesia in neuropathic rats. NeuroReport 2012; 23:535-539.

4. Perret DM, Kim DS, Li KW, Sinavsky K, Newcomb RL, Miller JM, Luo ZD. Application of pulsed radiofrequency currents to rat dorsal root ganglia modulates nerve injury-induced tactile allodynia. Anesth Analg 2011; 113:610-616.

5. Vallejo R, Tilley DM, Williams J, Labak S, Aliaga L, Benyamin RM. Pulsed radiofrequency modulates pain regulatory gene expression along the nociceptive pathway. Pain Physician 2013; 16:E601-E613.

6. Li DY, Meng L, Ji N, Luo F. Effect of pulsed radiofrequency on rat sciatic nerve chronic constriction injury: A preliminary study. Chin Med J (Engl) 2015; 128:540-544.

7. Jarahi M, Sheibani V, Safakhah HA, Torkmandi $\mathrm{H}$, Rashidy-pour A. Effects of progesterone on neuropathic pain responses in an experimental animal model for peripheral neuropathy in the rat: A behavioral and electrophysiological study. Neuroscience 2014; 256:403-411.

8. Jia Z, Ren H, Li Q, Ji N, Luo F. Pulsed radiofrequency reduced neuropathic pain behavior in rats associated with upregulation of GDNF expression. Pain Physician 2016; 19:49-58.

9. Henderson CE, Phillips HS, Pollock
RA, Davies AM, Lemeulle C, Armanini MP, Simmons L, Moffet B, Vandlen RA, Simpson LC corrected to Simmons L, Koliatsos VE, Rosenthal A. GDNF: A potent survival factor for motoneurons present in peripheral nerve and muscle. Science 1994; 266:1062-1064.

10. Buj-Bello A, Buchman VL, Horton A, Rosenthal A, Davies AM. GDNF is an age-specific survival factor for sensory and autonomic neurons. Neuron 1995; 15:821-828.

11. Bennett GJ, Xie YK. A peripheral mononeuropathy in rats that produces disorders of pain sensation like those in man. Pain 1988; 33:87-107.

12. Geng SJ, Liao FF, Dang WH, Ding X, Liu XD, Cai J, Han JS, Wan Y, Xing GG. Contribution of the spinal cord BDNF to the development of neuropathic pain by activation of the $\mathrm{NR}_{2} \mathrm{~B}$-containing NMDA receptors in rats with spinal nerve ligation. Exp Neurol 2010; 222:256-266.

13. Dixon WJ. Efficient analysis of experimental observations. Annu Rev Pharmacol Toxicol 1980; 20:441-462.

14. Lin ML, Lin WT, Huang RY, Chen TC, Huang SH, Chang $\mathrm{CH}$, Tsai SY, Chiu HW, Yeh GC, Lin CW, Wen YR. Pulsed radiofrequency inhibited activation of spinal mitogen-activated protein kinases and ameliorated early neuropathic pain in rats. Eur J Pain 2014; 18:659-670.

15. Park HW, Ahn SH, Son JY, Kim SJ, Hwang SJ, Cho YW, Lee DG. Pulsed radiofrequency application reduced mechanical hypersensitivity and microglial expression in neuropathic pain model. Pain Med 2012; 13:1227-1234.

16. Lin LF, Doherty DH, Lile JD, Bektesh S,
Collins F. GDNF: A glial cell line-derived neurotrophic factor for midbrain dopaminergic neurons. Science 1993; 260:1130-1132.

17. Ramer MS, Bradbury EJ, Michael GJ, Lever IJ, McMahon SB. Glial cell line-derived neurotrophic factor increases calcitonin gene-related peptide immunoreactivity in sensory and motoneurons in vivo. Eur ] Neurosci 2003; 18:2713-2721.

18. Boucher TJ, Okuse K, Bennett DL, Munson JB, Wood JN, McMahon SB. Potent analgesic effects of GDNF in neuropathic pain states. Science 2000; 290:124-127.

19. Höke A, Cheng C, Zochodne DW. Expression of glial cell line-derived neurotrophic factor family of growth factors in peripheral nerve injury in rats. Neuroreport 2000; 11:1651-1654.

20. Widenfalk J, Lundströmer K, Jubran $M$, Brene S, Olson L. Neurotrophic factors and receptors in the immature and adult spinal cord after mechanical injury or kainic acid. Journal of Neuroscience 2001; 21:3457-3475.

21. Shi JY, Liu GS, Liu LF, Kuo SM, Ton CH, Wen ZH, Tee R, Chen $\mathrm{CH}$, Huang $\mathrm{HT}$, Chen $\mathrm{CL}$, Chao D, Tai MH. Glial cell line-derived neurotrophic factor gene transfer exerts protective effect on axons in sciatic nerve following constrictioninduced peripheral nerve injury. Hum Gene Ther 2011; 22:721-731.

22. Sakai A, Asada M, Seno N, Suzuki H. Involvement of neural cell adhesion molecule signaling in glial cell line-derived neurotrophic factor-induced analgesia in a rat model of neuropathic pain. Pain 2008; 137:378-388. 